novelties such as the construction of a $T$ with an arbitrarily given curve as an $F$-curve of the first kind.

In the chapter on the resolution of singularities of surfaces the author does not give complete proofs but refers on essential points to the sources. There is indeed difference of opinion as to whether such resolution has actually been accomplished. Thus the present exposition of progress to date is opportune.

References to the bibliography are made in the text by number. This convenient method would have been much improved if the authors cited were also given.

A forthcoming report on topics in algebraic geometry contains chapters on Cremona transformations which will cover as well the matters not discussed by Miss Hudson. Fortunately the prior appearance of this volume has enabled the authors concerned both to make their general list of references more complete, and to refer for a broader account to the book itself.

A. B. COBLE

\title{
JORDAN ON STATISTICS
}

Statistique Mathématique. By Charles Jordan. Paris, Gauthier-Villars, 1927. xvii +344 pp.

This book is a scholarly treatment of the subject of mathematical statistics. It is in many respects the book which mathematicians have been waiting for. It performs for statistics much the same service as a standard Cours d'Analyse performs for analysis, and with the same typical French clearness; it would seem to be also of about the same degree of difficulty. Moreover, in point of difficulty, it is, considering the variety of subjects treated, of remarkably uniform grade throughout. The development is carefully planned in advance, and is carried out logically, and with uniform notation and nomenclature, producing maximum clearness of exposition in minimum space. There are no topics of dominant importance which the author has not considered, although there are some minor mattersthis is true of almost every book-which he has omitted and the reviewer would like to have had included. Apparently the author has also drawn on all the important sources, but though many times it is possible to recognize in his exposition the essential arguments of others, never are these arguments copied bodily; they always bear the impress of his own method of thinking. The only possible criticism in this connection is that, in acknowledging his indebtedness to others, Jordan rather frequently mentions names only, without giving references to the publications-a flaw which could easily be remedied in a second edition.

Although the various chapters are almost uniformly good, the reviewer was particularly impressed with the first three introductory chapters and with those on frequency curves ( 9 and 10) and less well pleased with the final chapter, on sampling. The first three chapters contain an excellent account of the preliminary notions; the material is well chosen and the sequence carefully planned. Much is left unproved here; the account is 
rather descriptive with only an occasional demonstration. Chapter 4, on probability, contains some stimulating material, including a rather elegant account of the series of Lexis and Poisson, along with much that is rather ordinary. The author is not always careful here to distinguish between what is really a definition and what is a theorem. The fundamental theorems which are hardest to demonstrate rigorously from his definition of probability, he does not prove at all, possibly because he makes reference to an account of probability which he has published elsewhere. His "empirical principle" is so vaguely stated that it would seem impossible to use it rigorously in a demonstration: (the italics are mine) "Si l'on répète une expérience un grand nombre de fois, chaque évènement se produit à peu près en un nombre de fois proportionnel à sa probabilité." On the next page (77) évènements indépendents are not defined at all. Assuming the author's definition of probability on page 28 , his statement of Bayes' Theorem on page 79 is not really a theorem, but an extension of the meaning of the earlier definition. It is rather unfortunate to say that (p. 86) J. Bernoulli proved his celebrated theorem when his so-called demonstration was really so very bad. Jordan lays a good deal of stress on the usefulness of the normal law as an approximation, but omits the case where the approximation is specially good, viz.: when it is a question of summing an equal number of terms of a point binomial on both sides of the mean. The reviewer gravely doubts that (p.120) "experience proves that the (normal) law of errors is alwa ${ }_{j} s$ valid when the errors are small relative to the magnitudes measured." Jurdan's account of the method of least squares is too compressed to be of mucn service to the reader. It follows classic lines, as do his directions for computation, and so he implies that he is not expecting the computer to use a machine!

Chapter 5, on Classification Statistique, is quite similar to Yule's treatment, but enjoys distinction from Yule's in not being verbose. Averages and moments are treated in the sixth chapter. Again there is a refreshing absence of that elaboration of the obvious which is characteristic of so many books at this point. Some of the illustrative material in this chapter is very interesting. Chapter 7 is on the point binomial. Chapters 9 and 10 are on frequency curves, and as has been indicated above, they seem to the reviewer excellent. He does not entirely agree with what appears to be the author's point of view (p. 207) as to the purpose of obtaining these analytical curves, namely, that it is solely to find a background of probability theory which one can then claim determines the data; but, adopting it for the moment, it would appear unfortunate not to have the "differentes considérations" (p. 241) which underlay Pearson's differential equation explained. For it is that very background of probability which has given Pearson's curves a theoretical advantage over certain other methods of representation. Jordan is, however, consistent when he criticizes Charlier's series and some others because they give negative frequencies. But the very small negative frequencies which he condemns are nevertheless very good approximations to the very small positive frequencies that they are supposed to equal, and so if one will admit that an important purpose of such series is to substitute for un wieldy expressions simple and manageable 
approximative formulas, that particular criticism is answered. Chapter 10 , on certain series fitted to frequency distributions by least squares, contains valuable suggestive material. Graduation, in Chapter 11, is treated rather curtly, but it was clearly necessary to avoid long incursions into special fields of application, and therefore it was probably necessary for Jordan to stop where he did or else so to extend this chapter that it would become out of harmony with the others.

Finally, we have the theories of correlation in Chapter 12, and of sampling in Chapter 13. This last subject involves matters of extreme delicacy, and they cannot be adequately set forth in a brief ten pages, even though all the statements be technically accurate. It is too easy here to confound approximations with exact statements, and indeed it is difficult to be sure that so-called approximations are not really wide of the mark. One such approximation which the author fails to indicate as such is " $\delta \mu_{2}=2 \zeta \delta \zeta \mathrm{m}$," used to find the probable error of the standard deviation when the probable error of the square of the standard deviation is given. Formula (15), which precedes the above equation, is also open to suspicion without the extra terms which have just been dropped. It is of course quite true if one admits the hypothesis that " $\eta=0$," but this condition is not usually satisfied in the many cases to which the formula and its derivatives are usually applied. Jordan seems to be aware of this himself but does not properly warn the reader.

The book is bound only in paper, but the printing and materials are good. The proof reading has been almost perfect. Only a few misprints have been noted, and they are listed below; no one of them happens to be of such a character as to mislead the reader, except perhaps the one on page 49.

Page 3, line 12, for Chieff read Chief. Page 13, first line of footnote, for $n(u)_{n}$ read $n h(u)_{n}$. Page 36 , last line, the omitted numerator of the first fraction is $s$. Page 49 , in the equation between equation (16) and equation (17), replace 2 by -2 . Page 83 , line 6 from bottom, for $\left(\frac{6}{1}\right)$ read $\left(\begin{array}{l}6 \\ 1\end{array}\right)$. Page 92, line 11, for 3, read 13. Page 241, line 12 from bottom: the omitted first letter should probably be L. Page 266: the upper limit is omitted in the integral of (1); it should be plus infinity. Page 288 , first line of footnote, for adjustement, read adjustment. The page following page 298 should be numbered 299, instead of 29 .

B. H. CAMP 